\title{
Nominalización en Procesos de Condensación de Significados en la Enseñanza de Solución Saturada: un estudio de caso en una escuela secundaria
}

\section{Nominalization in Processes of Condensation of Meanings in the Teaching of Saturated Solution: a case study in a secondary school}

D Guillermo Eduardo Cutrera'

D Marta Beatriz Massa ${ }^{2}$

Silvia Stipcich ${ }^{3}$

\begin{abstract}
'Universidad Nacional de Mar del Plata, Facultad de Ciencias Exactas y Naturales, Departamento de Educación Científica, Mar del Plata, Buenos Aires, Argentina. Autor correspondiente: gcutrera@mdp.edu.ar

${ }^{2}$ Universidad Nacional de Rosario, Facultad de Ciencias Exactas, Ingeniería y Agrimensura, Rosario, Santa Fé, Argentina.

${ }^{3}$ Universidad Nacional del Centro de la Provincia de Buenos Aires, Facultad de Ciencias Exactas y Naturales, Tandil, Buenos Aires, Argentina.
\end{abstract}

Resumen: En el trabajo didáctico con los términos propios de un campo disciplinar, uno de los recursos más frecuentes es el uso recurrente de sustantivos en la estrategia de nominalización. Tal nominalización implica una condensación de significados. En este trabajo se comunican los movimientos de empaque y desempaque de significados identificados y los recursos lingüísticos utilizados por una futura profesora de química durante el trabajo didáctico para construir el concepto de solución saturada. El tramo discursivo analizado corresponde a una clase que aborda la temática soluciones en su período de residencia docente en un aula de la educación secundaria. La investigación es de corte cualitativo, centrado en un estudio de caso que se enmarca en las nociones de nominalización y formas congruentes para estudiar los procesos de condensación de significados durante las interacciones discursivas. Se identifican los recursos lingüísticos que la practicante privilegia para la condensación de significados y se discuten sus implicaciones didácticas.

Palabras clave: Formación docente inicial; Discurso docente; Enseñanza de la química; Solución saturada.

Abstract: In teaching work with specific terminology of each field of discipline, the frequent use of nouns in the strategy of nominalization is one of the most common resources. Nominalization implies condensation of meanings. This paper communicates movements of packing and unpacking of meanings identified and linguistic resources used by a future Chemistry teacher during her didactic work to construct the concept of saturated solution. The discursive section that was analyzed corresponds to a class focused on the theme solutions during her residence period in a secondary school classroom. The research uses a qualitative approach and it is centered on a case study. It is framed in the notions of nominalization and congruent forms in order to analyze the processes of condensation of meanings during the discursive interactions. The linguistic resources for the condensation of meanings preferred by the future teacher are identified and its teaching implications are discussed.

Keywords: Pre-service teacher training; Teacher discourse; Chemistry teaching; Saturated solution.

Recebido em: 13/06/2020

Aprovado em: 21/02/2021 


\section{Introducción}

Diferentes investigaciones han estudiado las prácticas de enseñanza en aulas de ciencia desde una perspectiva discursiva (SERDER; JAKOBSSON, 2016; WALLACE, 2004). En estos contextos, la educación científica constituye una actividad lingüística específica o un encuentro entre diferentes discursos (KAMBERELIS; WEHUNT, 2012; WALLACE, 2004). Esta perspectiva sugiere que en la enseñanza en las aulas de ciencia convergen diferentes tipos de lenguajes que se expresan en un híbrido de idiomas (BAJTIN, 1934) o un discurso específico, en el que las expresiones científicas se mezclan con el uso del lenguaje coloquial o cotidiano. Este tipo de uso del lenguaje en la educación científica, con sus características lingüísticas y estructurales distintivas, también se ha descrito en el marco de la lingüística funcional sistémica (HALLIDAY; MATTHIESSEN, 2014). Varios estudios han demostrado que las demandas lingüísticas impuestas a los estudiantes a menudo van más allá de los desafíos conceptuales (SEAH; CLARKE; HART, 2011). En este contexto, aprender ciencia significa desarrollar una comprensión tanto conceptual como discursiva, que incluya las relaciones semánticas o los patrones temáticos específicos (LEMKE, 1997) y las características lingüísticas y estructurales distintivas del discurso científico.

Pero, ¿cómo actúa un futuro docente en sus experiencias iniciales en el aula para construir estructuras lingüísticas propias de la ciencia con sus significados? En este trabajo se identifican los movimientos de empaque y desempaque de significados y los recursos lingüísticos utilizados por una futura profesora de química durante el trabajo didáctico centrado en la temática 'soluciones'.

\section{Marco teórico}

Esta investigación propone una interpretación de la enseñanza de la ciencia en las aulas escolares considerando las perspectivas socioconstructivista y sociosemiótica.

Desde la perspectiva socioconstructivista, se recupera la relación entre contextos sociales, históricos y culturales (ROGOFF, 1990) y la acción individual (WERTSCH, 1993). Esta relación puede abordarse analizando la acción mediada. El lenguaje puede conceptualizarse como un instrumento de mediación omnipresente y constituye un requisito previo para todos los restantes tipos de mediación por artefactos culturales. Esta perspectiva tiene su fundamento teórico en la ley genética general del desarrollo cultural y su énfasis en la internalización de las experiencias sociales mediadas por herramientas culturales (VYGOTSKY, 1993). De estas herramientas culturales, el lenguaje fue visto por Vygotsky como la de mayor importancia, entendido como la "herramienta de herramientas" (WELLS, 1994). La relevancia del lenguaje reside no solo en su papel como instrumento de las interacciones sociales (herramienta cultural) sino también en tanto instrumento de pensamiento verbal (herramienta cognitiva) en el individuo (VYGOTSKY, 1993). El conocimiento científico es visto como construido socialmente, validado y comunicado y el aprendizaje de las ciencias como un proceso de enculturación (MORTIMER; SCOTT, 2003). Para Driver et al. (1994), en el proceso de aprender ciencias, los alumnos internalizan las herramientas culturales, devenidas como herramientas cognitivas, para la creación de significados. Este proceso involucra la participación de 
estudiantes y docentes en actividades discursivas de las lecciones de ciencias a través de las cuales "los alumnos se socializan en las formas de conocimiento y prácticas de la ciencia escolar" (DRIVER et al., 1994, p. 11).

Desde la perspectiva sociosemiótica, el lenguaje es entendido como una herramienta esencial para la construcción del conocimiento del individuo. Todo aprendizaje, incluido el científico experimental, se lleva a cabo únicamente porque existe el lenguaje como instrumento semiótico. El lenguaje es la condición esencial del conocimiento; el proceso por el cual la experiencia se convierte en conocimiento (HALLIDAY, 1989). Al proponer que el desarrollo del lenguaje es aprender a significar y que el aprendizaje en todas sus formas se caracteriza por el proceso de dar sentido, Halliday (1989) sostiene, además, que aprender el lenguaje es la base de todo ser humano. En esta teoría basada en el lenguaje, propuso una triple perspectiva de aprendizaje: "[...] aprender el lenguaje, aprender a través del lenguaje, aprender sobre el lenguaje" (HALLIDAY, 1993, p. 112, traducción nuestra). Simultáneamente, mientras está aprendiendo ciencias a través del lenguaje, está aprendiendo el lenguaje de la ciencia escolar y sobre ella, aunque no necesariamente a un nivel consciente. Por lo tanto, aprender el lenguaje de la ciencia escolar no se reduce, solamente, a aprender el significado de los conceptos científicos, sino que también incluye la capacidad de "[...] controlar las formas y estructuras lingüísticas únicas que construyen y comunican principios, conocimientos y creencias científicas" (FANG, 2005, p. 337, traducción nuestra). Desde estas exigencias se interpelan, además, a las prácticas de enseñanza.

\section{Palabras, relaciones semánticas y densidad semántica}

Las características gramaticales, la importancia de las palabras y los patrones semánticos típicos (LEMKE, 1997) que constituyen los lenguajes científicos pueden crear dificultades en su uso para los estudiantes (FANG, 2006). Además de términos científicos específicos (como equilibrio químico, solubilidad, reacción química y electrones), hay varias palabras cotidianas que también pueden tener una definición y uso específicos en la ciencia (como calor, trabajo, fuerza y energía). Diferentes investigaciones muestran que el empleo de este tipo de palabras frecuentemente crean confusión para los estudiantes (BROWN; SPANG, 2008; FANG, 2005; KARLSSON; LARSSON; JAKOBSSON, 2020). Además, varias palabras típicas del sujeto (como referencia, constante, patrón y factor) pueden tener distintos significados en diferentes disciplinas, lo que aumenta aún más la complejidad (SERDER; JAKOBSSON, 2016). Por ejemplo, en un estudio sobre el uso del lenguaje en aulas de ciencias, Gyllenpalm y Wickman (2011) encontraron que los estudiantes utilizaban el término experimento relacionado con tarea de laboratorio y refería, principalmente a una actividad pedagógica en contraste con una metodología de investigación.

Estas consideraciones adquieren relevancia en las aulas de ciencias si se considera el uso situacional del lenguaje $y$, consecuentemente, sus variaciones según sea el texto informal/formal, concreto/abstracto, cotidiano/especializado y según quién es el destinatario y cómo se comunica el texto. De esta manera, los discursos en las aulas se desplazan en un continuo entre una comprensión cotidiana del mundo (uso del lenguaje cotidiano) y una relación más distante con el mundo (uso del lenguaje 
científico). Los lenguajes y textos utilizados en el campo de la ciencia se caracterizan por la densidad de información, abstracción y tecnicismo (MARKIC; CHILDS, 2016). Halliday y Webster (2004) argumentan que el uso de nominalizaciones y formas pasivas es una técnica gramatical funcional para permitir la tarea del lenguaje cotidiano. Pero para una nueva generación que va a aprender ciencias en la escuela, esto puede llevar a que el lenguaje de enseñanza se perciba como demasiado intensivo en información y abstracto $y$, por lo tanto, obtenga un rasgo de objetividad y autoridad que a menudo carece de agencia humana.

Halliday y Martin (1993) enfatizan que la dificultad para comprender el lenguaje de la ciencia no solo existe a nivel de las palabras. Un lenguaje técnico (tecnicismo) va más allá del uso de palabras individuales y términos específicos. Los términos científicos derivan su significado y valor de estar taxonómicamente organizados, lo que implica que las relaciones semánticas se vuelven centrales para comprender los conceptos de la ciencia (LEMKE, 1997). Los términos técnicos y las entidades abstractas son centrales en la producción de conocimiento científico; una forma esencial de construir palabras científicas es mediante el uso de metáforas gramaticales como las nominalizaciones (HALLIDAY; MARTIN, 1993).

La nominalización constituye una característica esencial del discurso científico e implica la sustantivación de procesos y propiedades (CINTO, 2009). La nominalización es el recurso más poderoso para crear metáforas gramaticales. A través de este dispositivo, los procesos (escritos congruentemente como verbos) y las propiedades (escritas congruentemente como adjetivos) se reescriben metafóricamente como sustantivos (HALLIDAY; MATTHIESSEN, 2014). En la versión nominalizada, se pierde la información acerca de los participantes $\mathrm{y}$, normalmente, la de las circunstancias - empaque o condensación de la información -, de manera que la información que, congruentemente, se expresaría en una cláusula con sus componentes, se enuncia en forma resumida en un grupo nominal. Conceptualmente, se mantiene inmóvil a la realidad para permitir la observación y experimentación (HALLIDAY; MARTIN, 1993). AI proceso inverso (convertir la nominalización en una forma congruente) se le denomina desempaque (HALLIDAY; MATTHIESSEN, 2014). Esta densidad está relacionada con expresiones del lenguaje verbal con una alta proporción de palabras de contenido en las que la información se agrupa en nominalizaciones y grupos nominales extendidos (HALLIDAY; MARTIN, 1993).

Estos procesos son recuperados por Maton (2014a, 2014b) a partir de la noción de densidad semántica que se refiere al grado en que el significado se condensa en símbolos (términos, conceptos, frases, expresiones, gestos, etc.). Esta noción tiene similitudes con el término condensación (MATON; DORAN, 2017a). Cuanto más fuerte es la densidad semántica, mayor es la cantidad de significados que se condensan; cuanto más débil es la densidad semántica, menos significados se condensan. El grado de condensación dentro de una práctica sociocultural determinada se relaciona con la estructura semántica en la que se encuentra. Esta condensación de significados puede ser de carácter epistémico, esto es, puede referirse a conceptos o a descripciones empíricas o puede ser de carácter axiológico. Este trabajo se centra en la condensación epistémica de las definiciones formales y las descripciones empíricas (MATON, 2014a, 2014b, 2016), a partir de la noción de densidad semántica epistemológica (en adelante, densidad semántica). 


\section{Metodología}

La investigación realizada se inscribe en una metodología cualitativainterpretativa (GUBA; LINCOLN, 1994). En ésta, la fuente principal y directa de los datos son las situaciones naturales; los fenómenos se deben entender dentro de sus referencias espacio-temporales y su contexto (VALLES, 2000). Se recurrió a un estudio instrumental de casos (STAKE, 2013) que se propone la descripción de cómo se conforma el habla de futuros profesores de química en las situaciones de aula en que intervienen.

En Argentina, la formación del profesor en Química se realiza, tanto en instituciones de educación superior universitarias como no universitarias, en una carrera específica Profesorado de Educación en Química, con una duración de cuatro años. Si bien el Plan de Estudios puede diferir en su estructura curricular específica, se caracteriza por comprender espacios de formación en los campos de conocimiento propios de la química y de formación para la inserción ocupacional: trayecto sociohistórico-político, trayecto de formación pedagógico-didáctica y el trayecto de la práctica docente. Es en este último trayecto y finalizando la carrera, en el que se ubica la Residencia Docente como espacio curricular donde los futuros profesionales docentes planifican y desarrollan prácticas de enseñanza en cursos de instituciones de educación secundaria y superior. El presente estudio analiza las intervenciones discursivas de una practicante, que cursa la Residencia en el Profesorado en Química de la Universidad Nacional de Mar del Plata (Argentina), cuando Ileva adelante sus clases con estudiantes (14-15 años) en un aula de Fisicoquímica en un $2^{\circ}$ año de la educación secundaria de un colegio de la Provincia de Buenos Aires, Argentina. Fue seleccionado como el caso de estudio por cuanto el profesor responsable del curso le asignó a la practicante, explícitamente, la tarea de enseñar la construcción de explicaciones científicas escolares mientras aborda la unidad didáctica Disoluciones Químicas ubicada en el eje temático La naturaleza corpuscular de la materia de la propuesta curricular oficial. Este hecho convierte su discurso en el aula en peculiar y relevante para esta investigación.

La secuencia didáctica se desarrolló en un total de siete clases. En la primera de ellas se recuperaron las nociones de sistemas homogéneos, sustancia y soluciones. Durante la segunda y tercera clases, estudiantes y practicante trabajaron con la noción de concentración de las soluciones. En la clase siguiente los estudiantes resolvieron una actividad de evaluación y en la quinta clase se trabajó con las nociones de soluciones saturadas e insaturadas, comenzando a abordar la noción de solubilidad que se extendió durante las dos últimas clases en las que se trabajó con la dependencia del punto de saturación con la temperatura. Las clases fueron grabadas en audio y video y transcriptas en su totalidad.

Además, se registraron las reflexiones que la practicante explicita durante clases de discusión sobre el episodio que nos ocupa como pos-actividad en la asignatura Residencia. Este trabajo se focaliza en la primera parte de la quinta clase que se inicia con una actividad de mezcla de cloruro de sodio en agua a temperatura ambiente hasta formar una solución saturada ( $1^{\circ}$ episodio). Para el análisis, se recupera la división de la clase en episodios propuesta por la practicante quien utiliza como criterios tanto el 
cambio temático como el tipo de actividad desarrollado por los estudiantes (LEMKE, 1997), según los explicita en su diario de clase:

Para diferenciar los episodios de la clase, se tuvieron en cuenta los siguientes criterios: cambio en el tipo de recurso (por ejemplo, para reconocer el comienzo del segundo episodio tuve en cuenta que se comenzó a trabajar con un recurso didáctico diferente al que se estaba utilizando, que es el uso de un simulador virtual); cambios en el tipo de actividad (si el grupo de alumnos trabaja a partir de actividades de la guía, si se realizó corrección grupal, si realicé una experiencia demostrativa, etc.); cambio en la forma de diálogo (si se crea una situación de debate para sacar conclusiones sobre la experiencia, por ejemplo).

El episodio que se analiza en este artículo es aquel donde se trabajó con la caracterización de las soluciones según su clasificación en saturadas e insaturadas. Durante este episodio la practicante desarrolla tal clasificación y luego se centra en la noción de solubilidad. Para la diferenciación entre los tipos de soluciones utiliza un simulador como recurso didáctico.

Para el análisis de las participaciones discursivas se consideró la transcripción de la clase a texto completo y las reflexiones de la practicante sobre sus intervenciones durante el trabajo didáctico con la clasificación de las soluciones. Esto último permitió recuperar la voz de la practicante para seleccionar como fuentes aquellos elementos que ella destaca como significativos sobre sus intervenciones: "[...] tanto en el diálogo extraído de las desgrabaciones como en las diapositivas utilizadas como recurso durante la presentación del patrón temático" [D]'.

Durante un proceso de codificación abierta, se identificaron temas clave que permitieron entrever algunas categorías y subcategorías preliminares, desarrollando un análisis microscópico (o frase por frase) de los datos. El análisis del discurso de la practicante se realizó buscando indicadores que den cuenta de los procesos de empaque y desempaque implicados en la nominalización (organización de grupos nominales). Los fragmentos del discurso seleccionados, en función de tales indicadores, fueron asociados a categorías preliminares, proceso que permitió, por la recurrencia de significados observados en cada frase codificada, definir y describir finalmente cada una de las categorías y subcategorías.

Así se diferenció entre aquellos intercambios discursivos practicanteestudiantes en los cuales se priorizó el empleo de formas nominalizadas y aquellos en los que se priorizaron formas congruentes. Estas dos categorías - forma nominalizada y forma congruente - incluyen intervenciones de la practicante en las cuales refiere a solución saturada recurriendo a alguna de ambas modalidades. Los intercambios incluyeron intervenciones discursivas de la practicante durante las cuales recurrió a ambas formas inscribiéndolas en un proceso. Estas intervenciones son consideradas en la categoría denominada procesos que vinculan formas congruentes y nominalizadas bajo las subcategorías denominadas empaque y desempaque. Por otra parte, en la subcategoría desempaque se diferencian aquellos intercambios discursivos durante los cuales la practicante denota un referente empírico - generalmente en el contexto del trabajo con el simulador - de aquellas en las que, desempacando la información, no lo hace. Posteriormente, estas categorías y subcategorías fueron relacionadas entre sí delimitando la estructura jerárquica que se muestra en la ilustración 1.

${ }^{1} \mathrm{D}$ : documento de reflexión elaborado por practicante sobre sus intervenciones durante el trabajo didáctico con la clasificación de las soluciones. 
Ilustración 1 - Sistema de categorías elaborado durante el análisis

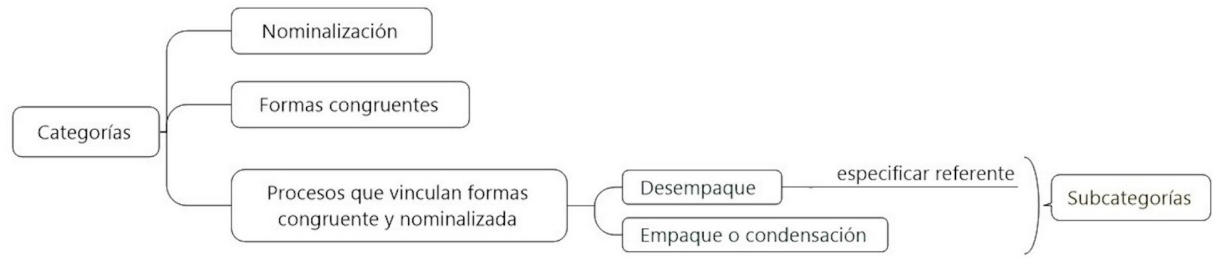

Fuente: elaborado por los autores.

Durante el análisis también se elaboraron marcas de nubes presentando las nueve palabras más frecuentes en las intervenciones discursivas de la practicante y se consideraron coincidencia con palabras derivadas y longitud mínima igual a cinco letras. El número de palabras se determinó a partir de un proceso de reducción por saturación de palabras relevantes.

\section{Análisis episódico}

En la primera parte del episodio la practicante recupera junto a los estudiantes la noción de solución saturada, abordada durante la clase anterior. Esta revisión es utilizada como introducción al trabajo con la noción de solubilidad. En su turno de habla la practicante refiere a la noción de solución saturada a partir de diferentes formulaciones, cada una de las cuales representa distintas aproximaciones al concepto.

En una primera intervención, la practicante recupera la noción de saturación centrando la atención en las cantidades relativas de soluto y de solvente, a efecto de diferenciar y establecer la condición de saturación. Focaliza su discurso en contrastar la idea de supera la cantidad de solvente con la de "el solvente que tengo no puede disolver más soluto que el que voy a ir agregando ¿sí?" (línea 12). 2,3 En esta intervención anticipa una conceptualización basada en las nociones de 'soluto' y de 'solvente' abstrayéndolas de la referencia contextualizada. Esta distinción, seguidamente, es reformulada por la practicante ("Entonces, como venían diciendo los chicos, el solvente ya no puede disolver más cantidad de soluto"; línea 12) y vinculándola con la noción de solución saturada ("Se tienen que acordar eso en las soluciones saturadas", línea 12). De esta forma, la condición establecida a partir de las cantidades relativas soluto/ solvente es empaquetada en el grupo nominal solución saturada. A continuación, la practicante presenta a este grupo como 'causa', inscribiéndolo en una estructura que le permite desempaquetar su significado ("Esto implica que se llega a un máximo valor de concentración", línea 12). En esta última intervención, además, se propone una nueva conceptualización a la noción de solución saturada introduciendo el término concentración trabajado en clases anteriores.

Durante estas intervenciones la practicante desarrolla movimientos discursivos centrados en torno a un grupo nominal - solución saturada - que implican procesos de nominalización y desempaque centrados en el empleo de términos del modelo

\footnotetext{
²ínea 12: corresponde a la línea de la transcripción correspondiente a la intervención.

${ }^{3}$ La disolución es un fenómeno de interacción soluto-disolvente y no una acción del disolvente sobre el soluto. No es correcto, por tanto, afirmar «el disolvente no disuelve más soluto». En este trabajo, el objeto del análisis es el discurso verbal de la practicante para identificar, en su organización, procesos de nominalización. Se destaca que, en función de los términos utilizados, el empaque puede incluir errores conceptuales.
} 
científico escolar trabajado en las clases anteriores. Utilizando un software como recurso didáctico, seguidamente, recupera un proceso simulado trabajado en clases anteriores con los estudiantes. La simulación permite visualizar el proceso de saturación de una solución a partir de la identificación perceptual de un depósito sólido en el fondo del recipiente. En este contexto, la practicante utiliza este criterio observacional como evidencia de saturación ("Habíamos visto en el simulador, no sé si se acuerdan, que una manera de reconocer a este tipo de soluciones, era cuando veíamos, por ahí está muy clarito pero, partículas de soluto depositadas en el fondo", línea 12). Esta intervención, caracterizada por una baja densidad semántica, precede a la nominalización ("¿Sí? entonces tenemos una solución saturada", línea 12) recuperando, finamente, el referente empírico simulado ("Observamos soluto depositado en el fondo", línea 12). En esta intervención, la practicante contextualiza y descontextualiza el contenido de su discurso utilizando un referente observable. Este proceso es analizado por la practicante, en su diario de clase, en términos de la transición entre niveles observable y conceptual -, proceso al que refiere como traducción. El primero de ellos alude al trabajo didáctico a partir de la percepción de un fenómeno; el segundo, a la conceptualización que puede interpretarse, siguiendo la propuesta de Taber (2013), en términos de los niveles de conceptualización macroscópico y submicroscópico: "Durante el desarrollo del patrón temático y el diálogo con los alumnos existe una traducción desde un nivel a otro. Al hablar de 'traducción' estoy haciendo referencia a la manera en que 'ubico' la respuesta de los alumnos, situada generalmente en un nivel observable, en una respuesta correspondiente al nivel conceptual" (D).

Durante los procesos de nominalización desarrollados por la practicante durante esta intervención discursiva, participantes - soluto y solvente -, proceso - disolver -, y circunstancias son eliminados del discurso, quedando los dos primeros condensados en el grupo nominal solución saturada. Cláusulas simples son comprimidas en un grupo nominal en la versión metafórica. En este proceso se desarrolla el empaque de la información. En particular, cuando refiere al reconocimiento de una solución saturada, recurriendo al criterio observable ("Habíamos visto en el simulador, no sé si se acuerdan, que una manera de reconocer a este tipo de soluciones, era cuando veíamos, por ahí está muy clarito pero, partículas de soluto depositadas en el fondo", línea 12), es posible reconocer la forma en la cual la practicante reconstruye la experiencia considerando a las partículas de soluto depositadas en el fondo del recipiente. En el cuadro 1 se representan los movimientos discursivos de la practicante durante una de sus participaciones (línea 12) en su evolución temporal. 
Cuadro 1 - Escala de dependencia contextual y condensación de significado para la noción solución saturada en la intervención de la practicante correspondiente a la línea 12

\begin{tabular}{|c|c|c|}
\hline \multicolumn{2}{|l|}{ + Dependencia contextual } & + Condensación de significados \\
\hline & $\begin{array}{l}\text { "[...] no es que supera la cantidad de } \\
\text { solvente sino que el solvente que tengo } \\
\text { no puede disolver más soluto que el que } \\
\text { voy a ir agregando. ¿Sí? [...]" }\end{array}$ & \\
\hline & $\begin{array}{l}\text { "Entonces, como venían diciendo los } \\
\text { chicos, el solvente ya no puede disolver } \\
\text { más cantidad de soluto. [...]" }\end{array}$ & \\
\hline & & $\begin{array}{l}\text { "[...] Se tienen que acordar eso en las } \\
\text { soluciones saturadas. [...]" }\end{array}$ \\
\hline & $\begin{array}{l}\text { "[...] Esto implica que se llega a un } \\
\text { máximo valor de concentración. [...]" }\end{array}$ & \\
\hline \multirow[t]{2}{*}{$\begin{array}{l}\text { "[...] Habíamos visto en el simulador, } \\
\text { no sé si se acuerdan, que una manera } \\
\text { de reconocer a este tipo de soluciones, } \\
\text { era cuando veíamos, por ahí está } \\
\text { muy clarito pero, partículas de soluto } \\
\text { depositadas en el fondo. [...]" }\end{array}$} & & \\
\hline & & $\begin{array}{l}\text { "[...] ¿Sí? entonces tenemos una solución } \\
\text { saturada. [...]" }\end{array}$ \\
\hline $\begin{array}{l}\text { "[...] Observamos soluto depositado en } \\
\text { el fondo [...]" }\end{array}$ & & \\
\hline
\end{tabular}

Fuente: elaborado por los autores.

Siguiendo la propuesta de Larsson (2018), se recupera e invierte el modelo de Maton (2014a), para ilustrar en el cuadro 1 las intervenciones de la practicante según la naturaleza de su contenido, en una escala horizontal, de izquierda a derecha, en lugar de verticalmente.

Estos movimientos constituyen una escala continua, sin límites exactos, entre un lenguaje cotidiano (izquierda) a un lenguaje científico escolar (derecha). Estas intervenciones son interpretadas según su mayor cercanía a un lenguaje contextual y cotidiano (izquierda) o a un lenguaje más condensado y específico (derecha). En algunos casos, las intervenciones discursivas de la practicante se ubican en la zona central del continuo, lo que puede interpretarse en términos del uso de un lenguaje intermedio (OLANDER, 2013) caracterizado por su carácter híbrido, en tanto constituido por términos pertenecientes a ambos lenguajes sociales. Las intervenciones discursivas de la practicante se ubican en alguna de estas instancias de enunciación; la sucesión temporal de estas intervenciones permite caracterizar los movimientos discursivos en términos de las transiciones entre enunciados que privilegian algunos de ambos lenguajes sociales o de aquellos que los incluyen simultáneamente.

Por otra parte, el continuo presentado en el cuadro 1 puede ser leído en términos de un aumento progresivo de la densidad semántica conforme se avanza del extremo representado por una mayor dependencia contextual hacia el de mayor condensación de significados. La inscripción temporal de las transiciones entre enunciaciones caracterizadas por estas modalidades de enunciados, define el perfil semántico del discurso en el aula, caracterizado en términos de su densidad semántica.

Al referir a la condensación epistemológica, Maton y Doran (2017a) distinguen diferentes tipos de herramientas. Entre ellas, la conexión ubica explícitamente significados dentro de constelaciones epistemológicas, por ejemplo, los esquemas 
clasificatorios. La clasificación describe a un término como un subtipo de otro: cada subtipo en la clasificación es un ejemplo del tipo. En un turno de habla posterior, en el mismo episodio, la practicante explicita la definición de soluciones insaturadas al desplegar un sistema clasificatorio que incluye a éstas junto a las soluciones saturadas. La aproximación conceptual a la noción de solución insaturada se realiza, por un lado, a partir de un proceso de metaforización, desde el contraste con solución saturada, recurriendo a una operación léxica - agregado del prefijo 'in' - a la nominalización previa: "[...] si decimos que en las saturadas nuestro solvente ya no puede disolver más soluto, en las insaturadas, por ende, o sea, por lógica, podemos suponer que sí admite más soluto. Todavía no llegó a la saturación ¿Lo ven eso?", línea 16. En esta última intervención, la practicante desempaqueta la noción de solución saturada, explicitando una significación que recupera en la caracterización para solución insaturada. La insaturación es significada tanto en términos de las cantidades relativas de soluto y solvente como a partir de la no-saturación.

En una nueva intervención el término solución insaturada es resignificado a partir de la noción de concentración. "Admitir más soluto" (línea 16) implica la posibilidad de modificar la concentración de la solución y esta idea aumenta el contenido informativo del término solución insaturada ("[...] A medida que vas agregando más soluto, se va concentrando la solución, ¿sí? [...]", línea 30). Sucesivamente, la posibilidad de aumentar la cantidad de soluto y el consiguiente aumento de la concentración, permiten aumentar la densidad semántica del término solución insaturada en sendos procesos de desempaque conceptual. La nominalización inicial, conducente a solución saturada es, seguidamente, des-andada durante la delimitación conceptual de solución insaturada. En esta misma intervención discursiva, la practicante fija la atención de los estudiantes en un referente empírico - como es el soluto depositado en el fondo del recipiente, presentado como indicador perceptual de la ausencia de saturación -, y finaliza su intervención expresando el proceso metafóricamente mediante un sustantivo ("[...] ¿sí? [...] no llega al punto de saturación, sí, porque no hay soluto depositado en el fondo. Bien [...]", línea 30).

Los términos saturada e insaturada aumentan la densidad semántica de la palabra solución (MATON, 2016), permitiendo una mayor diferenciación al explicitar el tipo (solución) en subtipos (saturada, insaturada). Los significados asociados con el tipo de orden superior son necesariamente adoptados por el subtipo de orden inferior (aunque no al revés) porque para que $X$ sea un subtipo de $Y$ debe poseer las características relevantes de $Y$ (además de sus propias características distintivas). Así se crean significados cruzados permitiendo un aumento de la densidad semántica tanto del término-tipo como de cada término-subtipo.

El despliegue de estas clasificaciones durante las intervenciones discursivas es recuperado por la practicante en una nueva instancia, centrada en la soluciones insaturadas ("Si tienen dudas, me frenan, pero dentro de las insaturadas vamos a clasificar a las soluciones, ¿tienen alguna duda? Pregunten [...] Bueno, dentro de las insaturadas podemos clasificar a las soluciones en diluidas y concentradas, ¿Les suenan esos términos?", línea 18). Recurre a la concentración, una propiedad de la solución para delimitar la diferenciación de cada término-subtipo ("[...] Va a estar concentrada porque tiene una concentración alta, podemos llamarlo así, y diluida, cuando está lejana a ese valor máximo. Es decir, que tiene poca cantidad de soluto [...]", línea 26) y al valor 
máximo de concentración definido para la solución saturada ("[...] Concentrada va a ser cuando esté bastante cerca de ese valor máximo, cuando esté cerca de saturarse ¿lo ven?", línea 26). Luego recupera ambos subtipos de solución insaturada a partir de una cláusula congruente seguida de una nominalización ("A medida que vas agregando más soluto, se va concentrando la solución, ¿sí? Todavía no llega al punto de saturación, sí, porque no hay soluto depositado en el fondo. Bien ¿Alguna duda hasta acá?", línea 30).

En la nube de palabras mostrada en la ilustración 2 se presentan las palabras más frecuentes durante las interacciones discursivas. Se priorizaron palabras técnicas para el patrón temático propuesto por la practicante y otras asumidas como relevantes durante el análisis de sus intervenciones. Entre estas últimas, fondo y máximo son utilizadas por la practicante para contextualizar los procesos nominalizados y diferenciar los tipos de soluciones, respectivamente.

llustración 2 - Marca de nube correspondiente a las palabras más frecuentes en las intervenciones discursivas de la practicante

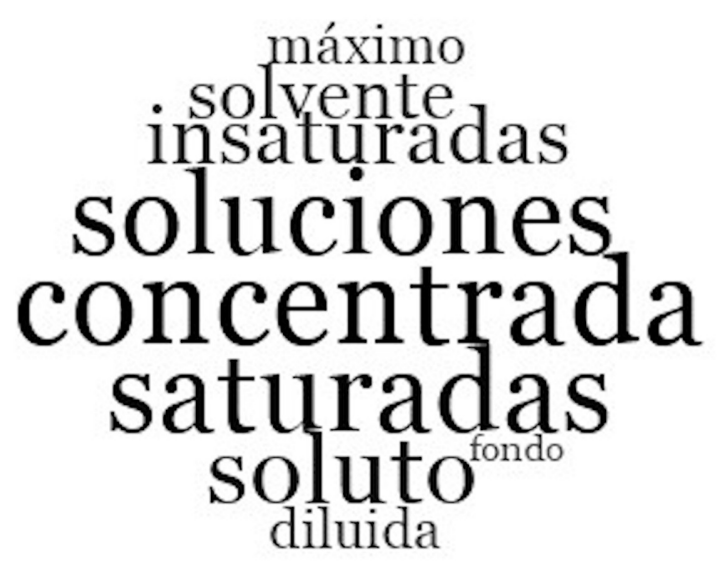

Fuente: elaborada por los autores.

Un término técnico es, frecuentemente, parte de una compleja red de significados que define su alcance conceptual. Los procesos de empaque y desempaque desarrollados por la practicante delimitan diferentes aproximaciones conceptuales para los grupos nominales propuestos durante este episodio. La practicante reconoce estas diferencias para el término solución saturada: "Se definió al término [solución saturada] a partir de diferentes relaciones semánticas. Lo mismo ocurrió con el concepto de solución insaturada. La siguiente tabla [cuadro 2, elaborado por la practicante], resume las relaciones semánticas que involucran a cada uno de estos conceptos".

Cuadro 2 - Diferentes relaciones semánticas utilizadas durante el trabajo discursivo con la noción de solución saturada*

\begin{tabular}{|l|l|}
\hline \multicolumn{1}{|c|}{ Concepto } & \multicolumn{1}{c|}{ Relaciones semánticas que involucran al término } \\
\hline Solución saturada & $\begin{array}{l}\text { [si se le agrega más] cantidad de soluto [no se modifica su] concentración } \\
\text { [llegan a un] máximo valor de concentración } \\
\text { [aquellas en que] el solvente [ya no puede disolver más] cantidad de soluto } \\
\text { [su] concentración [equivale al] valor de solubilidad } \\
\text { [podemos observar] soluto depositado en el fondo } \\
\text { [su] color [es muy] fuerte }\end{array}$ \\
\hline
\end{tabular}


Estas aproximaciones se diferencian entre sí en tanto suponen diferentes niveles de abstracción y su reconocimiento permite dar cuenta de las formas en que los significados se condensan en términos tales como solución saturada, solución insaturada, solución diluida y solución concentrada durante los intercambios discursivos practicante-estudiantes. Estas nominalizaciones delimitan condensaciones léxicas de significado, codificadas en un grupo nominal y constituyen un recurso lingüístico clave para explicar algunos fenómenos asociados a la construcción de conocimiento en las aulas de ciencias. La fortaleza de la densidad semántica no es intrínseca a una palabra, sino que depende de la constelación epistemológica de significados con los que se relaciona la palabra. Cuanto mayor sea la cantidad de relaciones establecidas con otros significados, más fuerte es la densidad semántica asociada a cada uno de estos grupos nominales. Estas relaciones presentadas por la practicante son diversas delimitando diferentes significados a cada uno de estos grupos; la misma palabra se puede utilizar como técnica o cotidiana. Esto es particularmente importante para la temática trabajada en este episodio considerando que algunos de los términos del patrón temático de la practicante resuenan en el lenguaje cotidiano de los estudiantes. En la ilustración 3 se muestran las diferentes conceptualizaciones empaquetadas en cada uno de estos grupos nominales.

Ilustración 3 - Conceptualizaciones incluidas en diferentes grupos nominales propuestas por la practicante en sus intervenciones discursivas*

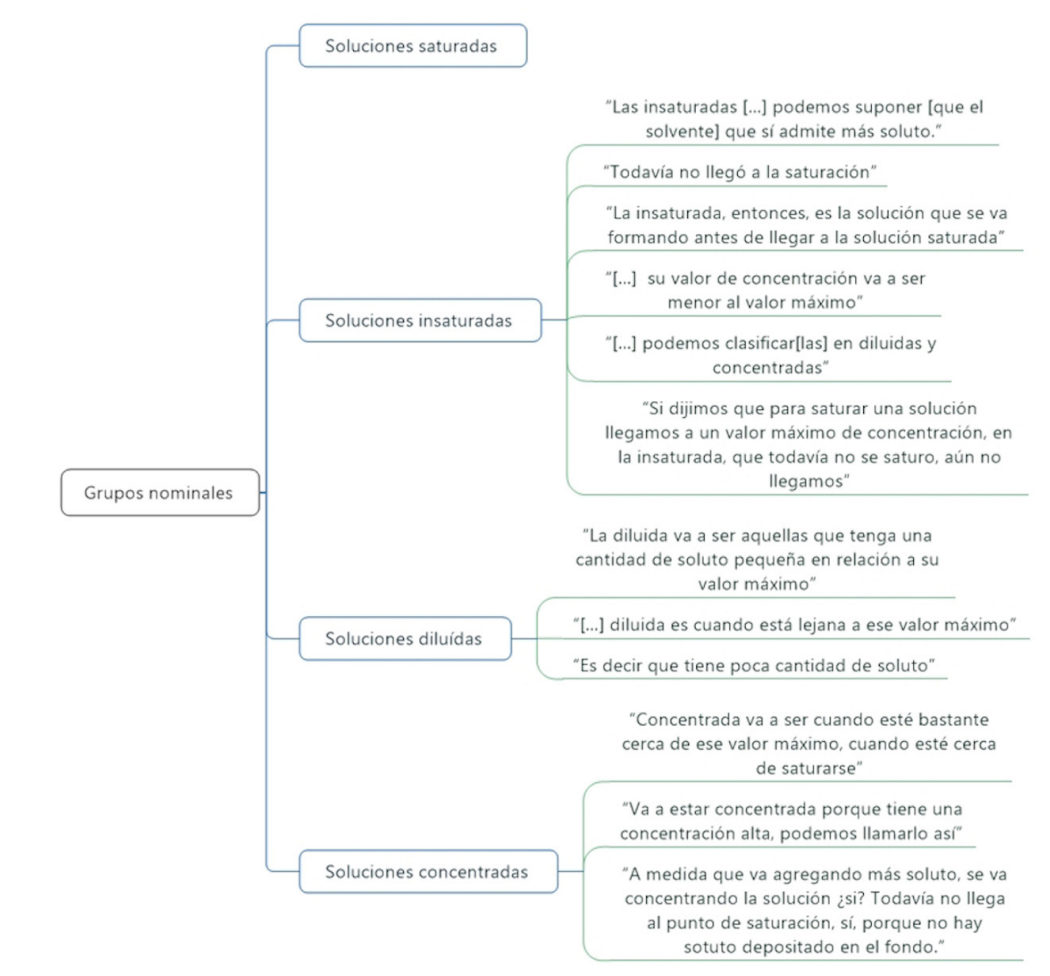

*Las citas para solución saturada se muestran en el cuadro 2.

Fuente: elaborado por los autores.

La practicante interpretó sus intervenciones en términos del trabajo didáctico entre los niveles observable y conceptual. En una reflexión general acerca del lenguaje utilizado y en referencia a los niveles con los que trabajó durante el episodio, reconoce, en su reflexión, la prevalencia del conceptual: "[...] en cuanto a los niveles de representación de la materia utilizados en la presentación del patrón temático se evidencia el privilegio en el uso del nivel conceptual por sobre el nivel observable" (D). 
A partir del análisis de la transcripción de la clase la residente reconoció las diversas aproximaciones conceptuales a la noción de solución saturada, por ejemplo, y lo expresó en los siguientes términos: "Al analizar las maneras de presentar estos términos durante el desarrollo del patrón temático se observó, en ambos casos, que cada uno de estos términos estaba involucrado en una importante cantidad de relaciones semánticas, que ofrecía diversidad en su conceptualización. Esta diversidad puede ser un factor de dificultad para el alumno en la comprensión de estos conceptos." (D).

En el análisis del episodio, la practicante interpreta las conceptualizaciones sobre solución saturada mostradas en el cuadro 2, en términos de la siguiente clasificación:

Del análisis de las relaciones semánticas que involucran al término soluciones saturadas se infiere la diversidad en las relaciones semánticas que incluyen a este concepto: éste es presentado a partir de la definición del mismo ('aquellas en que el solvente ya no puede disolver más cantidad de soluto'), de sus características ('llegan a un máximo valor de concentración') y utilizando relaciones semánticas con los términos: concentración, soluto y solvente. No necesariamente se utilizaron estos tres términos al mismo tiempo durante su definición ('si se le agrega más cantidad de soluto no se modifica su concentración'). Este término también es presentado a partir de sus variables observables ('podemos observar soluto depositado en el fondo'; 'su color es muy fuerte'). (D).

Además de explicitar que este último término "[...] estaba involucrado en una importante cantidad de relaciones semánticas que ofrecía diversidad en su conceptualización [...]", la practicante reconoce que "esta diversidad puede ser un factor de dificultad para el alumno en la comprensión de estos conceptos" (D). La diversidad de relaciones semánticas en la que se ubica la noción de solución saturada expresa, en la perspectiva de la practicante, el trabajo con el patrón temático. En particular, destaca que "[...] la complejidad indicada se acentúa con el hecho de estar relacionado con el término 'concentración' (que suele ser un término difícil de asimilar)" (D). Esta lectura de la residente, por un lado, expresa la dificultad conceptual que presenta, para los estudiantes, la noción de solución saturada considerando su conceptualización relacionada a la noción de concentración. Esta última nominalización compacta una red de significados que abstraen la noción de solución saturada. Pero, además, la noción de concentración priorizada por el grupo de estudiantes, estaría vinculada, según la practicante, a variables con referentes observables que, finalmente, fueron priorizados por los estudiantes: "Esto podría deberse al uso del simulador de concentración previo al desarrollo del concepto de 'soluciones saturadas' ya que en el mismo se trabaja con variables del tipo observables: presencia de soluto depositado en el fondo y cambio en el color de las soluciones a medida que aumenta la concentración de las mismas". (D)

La lectura de la noción de concentración, ligada a aspectos observables de la solución, expresaría tanto una forma privilegiada de significar el fenómeno por parte de los estudiantes como de las dificultades que les presentó la conceptualización asociada al patrón temático propuesto por la practicante. Según la residente, estas dificultades vinculadas a la conceptualización se expresaron en la persistencia de una fuerte referencia al contexto, expresada en las intervenciones discursivas de los estudiantes ("A pesar de la prevalencia del nivel conceptual durante el desarrollo del patrón, se observó que los alumnos se aferran a las variables observables en el momento de definir o reconocer un concepto", D). En sus intervenciones, los estudiantes privilegiaron la lectura del fenómeno - definido por la formación de la solución - delimitándolo a partir de referentes observables frente a la lectura centrada en una conceptualización en el nivel macroscópico, promovida por la practicante. En este contexto, las formas 
congruentes y nominalizadas para hablar sobre el fenómeno coexistieron durante los intercambios.

\section{Consideraciones finales}

La investigación sobre las prácticas en el aula durante la residencia docente se instala como un requisito constitutivo para el análisis de las situaciones de enseñanza, las actuaciones del docente, las intervenciones de los estudiantes, las propuestas didácticas y los recursos utilizados (STENHOUSE, 1998). En este contexto se enmarca el estudio de los recursos lingüísticos y la densidad semántica para analizar la manera en que se organiza la nominalización de solución saturada en el discurso de la practicante durante el episodio presentado. Los resultados permiten establecer algunos aspectos a tener en cuenta para la formación de futuros profesores en Química.

Las nominalizaciones constituyen condensaciones léxicas de significado codificadas en un grupo nominal, y representan un recurso lingüístico clave para explicar fenómenos fisicoquímicos. En este estudio se observa que el lenguaje utilizado por la practicante se caracteriza por priorizar el empleo de términos técnicos que denotan procesos que se expresan metafóricamente mediante sustantivos. Con menor frecuencia, la practicante recurre al empleo de enunciados más familiares y a un lenguaje no especializado, indicado por la observación de fenómenos cotidianos que recupera de la clase anterior.

La diversidad registrada en las conceptualizaciones propuestas por la practicante, se expresa en procesos que implican agrupamientos diferentes en un mismo grupo nominal que, si bien se homologa discursivamente a un mismo término, es provisto de una importante polisemia en tanto resume diferentes palabras y relaciones semánticas (como se observa en la ilustración 2). Este resultado permite reflexionar, durante la residencia docente, en instancias post-activas, acerca de la manera en que la misma etiqueta empleada en el grupo nominal expresa diferentes aproximaciones al concepto. Estas aproximaciones pueden permanecer atomizadas entre sí en tanto el docente no establezca explícitamente los nexos conceptuales pertinentes. En este contexto se instala la necesidad de desandar el proceso de nominalización disolviendo la alta densidad semántica concentrada en el grupo nominal y seleccionar adecuadamente qué conceptualizaciones han de ser privilegiadas.

Otro aspecto que se deriva de este estudio atiende a la orientación para la construcción de significados en el discurso científico escolar. La noción de solución, durante el episodio de esta clase fue resignificada conceptualmente a partir de la clasificación propuesta por la practicante. La clasificación permitió aumentar la densidad semántica del término solución tanto por las relaciones tipo/subtipo involucradas (MATON; DORAN, 2017b) como por la conceptualización propuesta para cada subtipo. En este último caso, el aumento en la densidad semántica se presentó con la diversidad inherente a las diversas aproximaciones conceptuales abordadas.

La tecnicidad, la nominalización y la clasificación se presentaron como recursos lingüísticos privilegiados por la practicante para la condensación de significados y para aumentar la densidad semántica. Se trata de recursos que posibilitan la condensación epistemológica (MATON; DORAN, 2017a). Como sostienen Chamorro, Mizuno y Moss (2003), con relación a los aprendizajes de los estudiantes, existe un factor 
clave en el manejo de las nominalizaciones: la concientización. En este trabajo se extiende esta importancia a la formación profesional docente: aprender a reconocer las nominalizaciones como procesos dinámicos, utilizados en sus discursos en el aula de ciencia. Esto es, además del reconocimiento de los procesos de empaque y desempaque, la necesidad de visibilizar que existe otro factor clave en el manejo de estos procesos: la toma de conciencia. En este contexto, Cinto (2009) desarrolla sugerencias para el manejo adecuado de las metáforas en el contexto pedagógico que, si bien fueron propuestas para el trabajo didáctico con textos escritos, pueden ser recuperadas en el contexto de las intervenciones discursivas docente. Entre ellas, des-ocultar la información presente en la nominalizaciones se presenta como particularmente valiosas en términos de des-naturalizar el empleo de estrategias discursivas tendientes a aumentar la densidad semántica en el discurso docente. Estas modalidades de intervención didáctica, además, favorecerían la movilidad discursiva (LARSSON; JAKOBSSON, 2017) entre los discursos cotidianos y científicos, así como entre significados concretos, abstractos, específicos y generales. En todo caso, el potencial didáctico tanto de esta movilidad como del empleo de nominalizaciones reside en la posibilidad de un trabajo reflexivo de parte del docente sobre sus intervenciones discursivas. En el contexto de la formación docente inicial, el análisis del propio discurso en el aula se presentó como un recurso didáctico valorado positivamente por la practicante, por ejemplo, en términos del reconocimiento de las dificultades didácticas durante la construcción de significados con los estudiantes ("El análisis realizado, centrado en el discurso, permite evidenciar estos niveles de dificultad", D). A partir de los resultados presentados en este trabajo se entiende que las instancias de reflexión centradas en identificar y explicitar los recursos lingüísticos utilizados durante la construcción de nominalizaciones se presentarían como un recurso privilegiado para la construcción del discurso docente en el aula de ciencia.

El aumento de la densidad semántica en el proceso de nominalización sin que se expliciten las relaciones entre las diferentes formas nominalizadas se presentaría como una fuente de dificultades potenciales para la conceptualización de los estudiantes. En este contexto se instala la necesidad de una mediación docente que explicite, en caso de presentarse, las relaciones entre las diferentes formas nominalizadas.

Finalmente, y recuperando recomendaciones dirigidas a sugerir que las prácticas de enseñanza de la ciencia deberían apelar más a la congruencia que a la metáfora gramatical en los niveles de escolaridad iniciales de la educación secundaria (CINTO, 2009), es interesante destacar el reconocimiento de la practicante a la tendencia de los estudiantes a ubicar los intercambios discursivos en referencia a eventos observables: Esta tendencia es justificada, en este estudio, por referencia al empleo de procesos simulados en clases anteriores: En este contexto, al ofrecer referentes empíricos explícitos, el trabajo didáctico con procesos simulados permitiría al docente recurrir al empleo de formas congruentes que facilitarían desocultar y mediar discursivamente en la construcción de formas nominalizadas.

\section{Referencias}

BAJTIN, M. La palabra en la novela. In: BAJTIN, M. Teoría y estética de la novela. Madrid: Taurus, 1934. p. 77-236. 
BROWN, B. A.; SPANG, E. Double talk: synthesizing everyday and science language in the classroom. Science Education, Hoboken, v. 92, n. 4, p. 708-732, 2008. DOI: https://doi.org/10.1002/ sce.20251.

CHAMORRO, D.; MIZUNO, J.; MOSS, G. Tergiversaciones y correspondencias: la metáfora y sus bemoles. Revista Latinoamericana de Estudios del Discurso, Caracas, v. 3, p. 29-47, 2003.

CINTO, M. T. La nominalización: obstáculo para la comprensión lectora. Educación, Lenguaje y Sociedad, La Pampa, v. 6, n. 6, p. 171-186, 2009.

DRIVER, R.; ASOKO, H.; LEACH, J.; SCOTT, P.; MORTIMER, E. Constructing scientific knowledge in the classroom. Educational Researcher, Washington, v. 23, n. 7, p. 5-12, 1994. DOI: https://doi. org/10.3102/0013189X023007005.

FANG, Z. Scientific literacy: a systemic functional linguistics perspective. Science Education, Hoboken, v. 89, n. 2, p. 335-347, 2005. DOI: https://doi.org/10.1002/sce.20050.

FANG, Z. The language demands of science reading in middle school. International Journal of Science Education, London, v. 28, n. 5, p. 491-520, 2006. DOI: https://doi. org/10.1080/09500690500339092.

GUBA, E. G.; LINCOLN, Y. S. Competing paradigms in qualitative research. In: DENZIN, N. K.; LINCOLN, Y. S. (ed.). Handbook of qualitative research, vol. 2. Thousand Oaks, CA: Sage, 1994. p. 105-117.

GYLLENPALM, J.; WICKMAN, P. O. "Experiments" and the inquiry emphasis conflation in science teacher education. Science Education, Hoboken, v. 95, n. 5, p. 908-926, 2011.

HALLIDAY, M. Some grammatical problems in scientific English. In: HALLIDAY, M. A. K.; MARTIN, J. R. (ed.). Writing science: literacy and discursive power. Washington: Falmer Press, 1989. p. 69-85.

HALLIDAY, M. Towards a language-based theory of learning. Linguistics and Education, New York, v. 5, n. 2, p. 93-116, 1993. DOI: https://doi.org/10.1016/0898-5898(93)90026-7.

HALLIDAY, M.; MARTIN, J. Writing science: literacy and discursive power. Washington: Falmer Press, 1993.

HALLIDAY, M.; MATTHIESSEN, C. An introduction to functional grammar. London: Routledge, 2014.

HALLIDAY, M.; WEBSTER, J. The language of science, vol. 5. London: Continuum International Publishing, 2004.

KAMBERELIS, G.; WEHUNT, M. D. Hybrid discourse practice and science learning. Cultural Studies of Science Education, Dordrecht, v. 7, n. 3, p. 505-534, 2012. DOI: https://doi.org/10.1007/s11422012-9395-1.

KARLSSON, A.; LARSSON, P. N.; JAKOBSSON, A. The continuity of learning in a translanguaging science classroom. Cultural Studies of Science Education, Dordrecht, v. 15, p. 1-25, 2020. DOI: https://doi.org/10.1007/s11422-019-09933-y.

LARSSON, P. N. "We're talking about mobility": discourse strategies for promoting disciplinary knowledge and language in educational contexts. Linguistics and Education, New York, v. 48, p. 61-75, 2018.

LARSSON, P. N.; JAKOBSSON, A. Semantiska vågor-elevers diskursiva rörlighet i gruppsamtal. Nordic Studies in Science Education, v. 13, n. 1, p. 17-35, 2017. DOI: https://doi.org/10.5617/ nordina.2739.

LEMKE, J. Aprender a hablar ciencia: lenguaje, aprendizaje y valores. Barcelona: Paidós, 1997. 
MARKIC, S.; CHILDS, P. E. Language and the teaching and learning of chemistry. Chemistry Education Research and Practice, London, v. 17, n. 3, p. 434-438, 2016.

DOI: https://doi.org/10.1039/C6RP90006B.

MATON, K. Building powerful knowledge: the significance of semantic waves. In: BARRETT, B.; RATA, E. (ed.). Knowledge and the future of the curriculum. London: Palgrave Macmillan, 2014a. p. 181-197.

MATON, K. Knowledge and knowers: towards a realist sociology of education. London: Routledge, 2014b.

MATON, K. Legitimation code theory: building knowledge about knowledge-building. In: HOOD, S.; SHAY, S. (ed.). Knowledge-building: educational studies in legitimation code theory. London: Routledge, 2016. p. 1-24.

MATON, K.; DORAN, Y. J. Condensation: a translation device for revealing complexity of knowledge practices in discourse, part 2 - clausing and sequencing. Onomázein, Chile, p. 77-110, 2017a. (Número especial SFL).

MATON, K.; DORAN, Y. J. Semantic density: A translation device for revealing complexity of knowledge practices in discourse, part 1 - wording. Onomázein, Chile, p. 46-76, 2017b. (Número especial SFL).

MORTIMER, E. F.; SCOTT, P. Meaning making in secondary science classrooms. Philadelphia: Open University Press, 2003.

OLANDER, C. Why am I learning evolution?: pointers towards enacted scientific literacy. Journal of Biological Education, New York, v. 47, n. 3, p. 175-181, 2013. DOI: https://doi.org/10.1080/00219 266.2013.821750.

ROGOFF, B. Apprenticeships in thinking: cognitive development in social context. Oxford, UK: Oxford University Press, 1990.

SEAH, L. H.; CLARKE, D. J.; HART, C. E. Understanding students' language use about expansion through analyzing their lexicogrammatical resources. Science Education, Hoboken, v. 95, n. 5, p. 852-876, 2011. DOI: https://doi.org/10.1002/sce.20448.

SERDER, M.; JAKOBSSON, A. Language games and meaning as used in student encounters with scientific literacy test items. Science Education, Hoboken, v. 100, n. 2, p. 321-343, 2016.

STAKE, R. E. Multiple case study analysis. New York: Guilford Press, 2013.

STENHOUSE, L. La investigación como base de la enseñanza. Madrid: Morata, 1998.

TABER, K. Revisiting the chemistry triplet: drawing upon the nature of chemical knowledge and the psychology of learning to inform chemistry education. Chemistry Education Research and Practice, London, v. 14, n. 2, p. 156-168, 2013. DOI: https://doi.org/10.1039/C3RP00012E.

VALLES, M. Técnicas cualitativas de investigación social: reflexión metodológica y práctica profesional. Madrid: Síntesis Ed., 2000.

VYGOTSKY, L. S. Pensamiento y lenguaje. In: VYGOTSKY, L. S. Obras escogidas: vol. II. Madrid: Visor, 1993. p. 11-348.

WALLACE, $C$. Framing new research in science literacy and language use: authenticity, multiple discourses, and the "third space". Science Education, Hoboken, v. 88, n. 6, p. 901-914, 2004.

WELLS, G. The complementary contributions of Halliday and Vygotsky to a "language-based theory of learning". Linguistics and Education, New York, v. 6, n. 1, p. 41-90, 1994.

WERTSCH, J. Voces de la mente: un enfoque sociocultural para el estudio de la acción mediada. Madrid: Visor, 1993. 